\title{
THE CARTAN DETERMINANT AND GENERALIZATIONS OF QUASIHEREDITARY RINGS
}

\author{
by W. D. BURGESS and K. R. FULLER
}

(Received 30th October 1995)

\begin{abstract}
The Cartan determinant conjecture for left artinian rings was verified for quasihereditary rings showing $\operatorname{det} C(R)=\operatorname{det} C(R / I)$, where $I$ is a projective ideal generated by a primitive idempotent. This article identifies classes of rings generalizing the quasihereditary ones, first by relaxing the "projective" condition on heredity ideals. These rings, called left $k$-hereditary are all of finite global dimension. Next a class of rings is defined which includes left serial rings of finite global dimension, quasihereditary and left 1-hereditary rings, but also rings of infinite global dimension. For such rings, the Cartan determinant conjecture is true, as is its converse. This is shown by matrix reduction. Examples compare and contrast these rings with other known families and a recipe is given for constructing them.
\end{abstract}

1991 Mathematics subject classification: 16E10, 16P20.

\section{Introduction}

Let us recall that the Cartan determinant conjecture asserts that if $R$ is a left artinian ring with $\mathrm{gl} \operatorname{dim} R<\infty$ then $\operatorname{det} C(R)=1$. (See the survey [6].) Among the various lines of attack on this conjecture there are two which involve matrix reduction. We suppose for the moment that $R$ is left artinian and $\mathrm{gl} \operatorname{dim} R<\infty$. The first method was initiated by Zacharia in [12] and was used in [4], [9] and [1]. Here the idea is to find $e^{2}=e \in R$ so that $\operatorname{det} C(R)=\operatorname{det} C(e R e)$ and $g l \operatorname{dim} e R e<\infty$. The second method is to find an ideal $I$ so that $\operatorname{det} C(R)=\operatorname{det} C(R / I)$ and $g l \operatorname{dim} R / I<\infty$. The easiest case is where $I=R e R$, for some idempotent $e$, is an heredity ideal (see, e.g., [3]). Generalizations of this method are found in [9] and [11].

Our approach is to develop further the matrix reduction method where $I=R e R, e$ a primitive idempotent, $p \operatorname{dim}_{R} I<\infty$, but $R$ is not necessarily of finite global dimension. This leads to the identification of two classes of rings which generalize the quasihereditary rings. The first, called left $k$-hereditary, are always of finite global dimension, with the global dimension bounded above by a bound generalizing that for quasihereditary rings. The second, called rings with left matrix reducing series, gives a class of rings for which the Cartan determinant conjecture and its converse hold. Rings of finite global dimension in this class include all left serial rings of finite global dimension, the left 1-hereditary and the quasihereditary rings. Examples are then presented which illustrate these classes. A method of constructing rings with left matrix reducing series from two given such rings is exhibited. 
Throughout $R$ will be a semiprimary ring with a basic orthogonal set of primitive idempotents idem $(R)=\left\{e_{1} \ldots, e_{n}\right\}$. The radical is denoted $J(R)$, or just $J$. The Loewy length of a module $M$ is LL (M). If $R$ is left artinian, the (left) Cartan matrix of $R$ will be denoted $C(R)=\left(c_{i j}\right)$.

This work was done while the first author was enjoying the hospitality of the University of Iowa; he also acknowledges partial support from grant A7539 of the NSERC.

\section{Definitions and preliminary results}

We begin by considering a class of rings of finite global dimension which contains the quasihereditary rings. Their defining condition is strong enough to yield a bound on that dimension which generalizes [5, Statement 9].

Proposition 1.1. Let $R$ be a semiprimary ring. Suppose $0=I_{0} \subset I_{1} \subset \cdots \subset I_{t}=R$ is a chain of idempotent ideals such that for $j=1, \ldots, t$,

(1) $I_{j} J I_{j} \subseteq I_{j-1}$; and

(2) $\mathrm{p} \operatorname{dim}\left({ }_{R / I_{j-1}} I_{j} / I_{j-1}\right)=l_{j}<\infty$.

Then $\operatorname{gl} \operatorname{dim} R \leq \Sigma_{j=1}^{t-1} l_{j}+2 t-2$.

Proof. We first get an estimate on projective dimension which will be applied to the simples of $R$.

Let $I$ be any ideal of $R$ and put $B=R / I$. If $\mathrm{p} \operatorname{dim}_{R} I=l<\infty$ and $M$ is a left $B$ module, then $\mathrm{p} \operatorname{dim}_{R} M \leq l+1+\mathrm{p} \operatorname{dim}_{B} M$. To see this, the exact sequence $0 \rightarrow_{R} I \rightarrow_{R} R \rightarrow_{R} B \rightarrow 0$ shows that $\mathrm{p} \operatorname{dim}_{R} B=l+1$. In particular, if ${ }_{B} P$ is projective then $\mathrm{p} \operatorname{dim}_{R} P \leq l+1$. Thus if

$$
0 \rightarrow P_{d} \rightarrow \cdots \rightarrow P_{1} \rightarrow P_{0} \rightarrow M \rightarrow 0
$$

is a projective resolution of $M$ over $B$, then $\mathrm{p} \operatorname{dim}_{R} M \leq d+l+1$.

The following notation will be used: for $j=0, \ldots, t-1, R_{j}=R / I_{j}$ and $I_{j+1} / I_{j}=\bar{I}_{j+1}$. Since $J\left(R_{t-1}\right)=0, R_{t-1}$ is semisimple. If $t=1$ we are done. Otherwise, assume for purpose of induction, that for $1 \leq j<t-1$ that the theorem is true for $R_{j+1}$. Let the bound on $g l \operatorname{dim} R_{j+1}$ given by the theorem be $d$. The simple $R_{j}$-modules annihilated by $\bar{I}_{j+1}$ are of projective dimension at most $l_{j}+1+d$, by the lemma. Pick $e^{2}=e \in R_{j}$ with $\vec{I}_{j+1}=R_{j} e R_{j}([5$, Statement 6]). Consider a presentation

$$
0 \rightarrow J\left(R_{j}\right) e \rightarrow R_{j} e \rightarrow R_{j} e / J\left(R_{j}\right) e \rightarrow 0 .
$$

Since $e J\left(R_{j}\right) e=0, J\left(R_{j}\right) e$ has composition factors only among the simples annihilated by $\bar{I}_{j+1}$. This means that $\mathrm{p} \operatorname{dim}_{R_{j}} R_{j} e / J\left(R_{j}\right) e \leq l_{j}+1+d+1$, and completes the induction. 
Note that if $R$ has a chain of ideals as in (1.1) then $L L(R) \leq 2^{t}-1$, just as in [5, Statement 9]. Notice also that if $R$ has such a chain, then the same chain satisfies the right hand version of the hypotheses, given the conclusion of (1.1). It is readily seen that the rings of (1.1) have no loops in their quivers.

Definition 1.2. Let $R$ be semiprimary and $k \geq 0$. We say $R$ is left $k$-hereditary whenever idem $(R)$ can be ordered $e_{1}, \ldots, e_{n}$, so that letting $I_{0}=0$ and $I_{j}=R\left(e_{1}+\cdots+e_{j}\right) R, i=1, \ldots, n$, we have

(1) $e_{j} J e_{j} \subseteq I_{j-1}$; and

(2) $\mathrm{p} \operatorname{dim}\left({ }_{R / I_{j-1}} I_{j} / I_{j-1}\right)=l_{j} \leq k$, for $j=1, \ldots, n$.

(The right hand version is defined similarly.)

Examples will show that "left $k$-hereditary" is not left-right symmetric. However, (1.1) shows that $R$ left $k$-hereditary implies $R$ right $l$-hereditary, for some $l$. Of course, "left 0 -hereditary" means " quasihereditary", which is a symmetric concept.

Corollary 1.3. If $R$ is left $k$-hereditary, using the notation of (1.2), then gl $\operatorname{dim} R \leq \sum_{j=1}^{n-1} l_{j}+2 n-2 \leq(n-1)(k+2)$.

The Cartan determinant conjecture was verified for a quasihereditary left artinian ring $R$ in [3, Proposition 3.1] by observing that since idem $(R)$ can be ordered $e_{1}, \ldots, e_{n}$ so that if $I_{0}=0, I_{j}=R\left(e_{1}+\cdots+e_{j}\right) R, j=1, \ldots, n$, then $I_{j} / I_{j-1}$ is projective over $R / I_{j-1}$ and $e_{j} J e_{j} \subseteq I_{j-1}$, it follows that $\operatorname{det} C(R)=\operatorname{det} C\left(R / I_{1}\right)=\cdots=\operatorname{det} C\left(R / I_{n-1}\right)=1$. In [1], generalizing quasihereditary, an algebra $R$ is called neat in case idem $(R)$ can be ordered $e_{1}, \ldots, e_{n}$ so that if $\varepsilon_{i}=e_{i}+e_{i+1}+\cdots+e_{n}$ then the terms in the $\varepsilon_{i} R \varepsilon_{i}$-minimal projective resolution of $\varepsilon_{i} J e_{i}$ all belong to add $\left(\varepsilon_{i} R \varepsilon_{i+1}\right)$; and the authors observed that, as in [9, Proposition 4], based on [12], $\operatorname{det} C(R)=\operatorname{det} C\left(\varepsilon_{1} R \varepsilon_{1}\right)=\cdots=C\left(\varepsilon_{n-1} R \varepsilon_{n-1}\right)=1$. These results suggest Definition 1.4, below.

The notions "left $k$-hereditary" and "neat" coincide for left serial rings (see (2.4)), but not for more general rings (Example 3.2). We shall see that if $R$ is left 1-hereditary then $\operatorname{det} C(R)=1$. The example in Section 3 of [3] is left 1-hereditary, using, for example, $e_{1}, e_{2}, e_{4}, e_{3}, e_{5}$ and right 1 -hereditary using $e_{4}, e_{5}, e_{3}, e_{2}, e_{1}$. It is of global dimension 3 and is not quasihereditary. (It is also neat via the ordering $e_{5}, e_{1}, e_{2}, e_{3}, e_{4}$.)

Definition 1.4. Let $R$ be left artinian. A primitive idempotent $e$, which may be taken in idem $(R)$, is called left a matrix reducing idempotent whenever idem $(R)$ may be ordered, $e=e_{1}, \ldots, e_{n}$ in such a way that

(1) $\mathrm{p} \operatorname{dim}{ }_{\mathrm{R}} R e_{1} R<\infty$, and

(2) for each $k>1, R e_{1} R e_{k}$ has a projective resolution with terms from add $R\left(e_{1}+\cdots+e_{k-1}\right)$. 
An ordering $e_{1}, \ldots, e_{n}$ of idem $(R)$ is called a left matrix reducing series if each $e_{j}+I_{j-1}$, for $j=1, \ldots, n-1$, is matrix reducing in $R / I_{j-1}$, where $I_{0}=0$ and $I_{j}=R\left(e_{1}+\cdots+e_{j}\right) R, j=1, \ldots, n$.

If $e_{1}, \ldots, e_{n}$ is a left matrix reducing series, then the 1,1 -entry of $C\left(R / I_{j-1}\right)$ is denoted $\tilde{c}_{j j}$

We shall soon see that every left 1 -hereditary ring and every left serial ring of finite global dimension has a matrix reducing series.

Lemma 1.5. If $R$ is left artinian and has a left matrix reducing idempotent $e_{1}$ then elementary column operations of adding a multiple of a column to a column strictly to its right will reduce the Cartan matrix $C(R)$ to the form

$$
\left(\begin{array}{cc}
c_{11} & 0 \cdots 0 \\
\vdots & C \\
c_{n 1} &
\end{array}\right),
$$

where $C=C\left(R / R e_{1} R\right)$. Moreover, $\operatorname{det} C(R)=c_{11} \operatorname{det} C\left(R / R e_{1} R\right)$.

Proof. Order idem $(R)$ as in (1.4). Consider a minimal projective resolution of $R e_{1} R e_{i}$. Each term of it is isomorphic to a direct sum of indecomposable projectives $R e_{j}$, where, by hypothesis, $j<i$. This says that the Cartan matrix (column) $C\left(R e_{1} R e_{i}\right)$ is a linear combination of the columns $C\left(R e_{j}\right), j<i$. Then the $i$ column of $C(R)$ can be converted into $C\left(R e_{i}\right)-C\left(R e_{1} R e_{i}\right)=C\left(R e_{i} / R e_{1} R e_{i}\right)$ by the column operations indicated.

Theorem 1.6. Let $R$ be a left artinian ring which has a left matrix reducing series, $e_{1}, \ldots, e_{n}$. Then

(a) $\operatorname{det} C(R)=\Pi_{i=1}^{n} \tilde{c}_{i j}$. In particular $\operatorname{det} C(R)>0$ and so if $\operatorname{gl} \operatorname{dim} R<\infty$ then $\operatorname{det} C(R)=1$. (i.e., the Cartan determinant conjecture is verified for these rings.)

(b) If $\operatorname{det} C(R)=1$ then $g l \operatorname{dim} R<\infty$. (i.e., the converse of the Cartan determinant conjecture is true for these rings.)

Proof. (a) This part follows immediately by successively applying (1.5). (b) In this case $R$ is left $k$-hereditary since the condition $\tilde{c}_{j j}=1$ is precisely the condition (1) of (1.1). Hence, $R$ has finite global dimension by (1.1).

We now look at the 1-hereditary case.

Proposition 1.7. Suppose $R$ is left artinian and has a primitive idempotent $e_{1}$ so that $e_{1} J e_{1}=0$ and $\mathrm{p} \operatorname{dim}_{R} R e_{1} R \leq 1$. Then $e_{1}$ is a left matrix reducing idempotent. 
Proof. We can number the idempotents so that if some $R e_{1} R e_{i}=0$, then $R e_{1} R e_{2}=\cdots=R e_{1} R e_{k}=0$ and $R e_{1} R e_{i} \neq 0$ for $i>k$. (The case where all $R e_{1} R e_{i}$ are projective is where $R e_{1} R$ is a heredity ideal.) If there is $k<i \leq n$ with $\mathrm{p} \operatorname{dim} R e_{1} R e_{i}=1$, suppose its minimal projective resolution has the form

$$
0 \rightarrow P \oplus R e_{j} \rightarrow R e_{1}^{(m)} \rightarrow R e_{1} R e_{i} \rightarrow 0,
$$

for some $j$. Since $e_{1} J e_{1}=0$, it follows that $1<j \leq k$. Hence, the ordering $e_{1}, \ldots, e_{k}, \ldots, e_{n}$ is left matrix reducing.

Corollary 1.8. If the left artinian ring $R$ is left 1-hereditary then it has a left matrix reducing series, and, hence, $\operatorname{det} C(R)=1$.

\section{Applications to left serial rings.}

In this section some of the ideas of the first section are explored for left serial rings. The following will be useful for building examples and for the reduction of the left serial case to where the (left) quiver is a cycle.

Proposition 2.1. Suppose $R$ is a left artinian ring which has orthogonal idempotents $E, F$ such that $E+F=1$ and $F R E=0$. Suppose further that $E R E$ is of finite global dimension and that ERE and $F R F$ have left matrix reducing series, $e_{1}, \ldots, e_{k}$ and $f_{1}, \ldots, f_{l}$, respectively. Then $e_{1}, \ldots, e_{k}, f_{1}, \ldots, f_{t}$ is a left matrix reducing series for $R$.

Proof. The methods of [6, Proposition 2.5] are used.

Since $e_{1}$ is a left reducing idempotent for $E R E$, changing labels, if necessary, we can assume that the ordering $e_{1}, \ldots, e_{k}$ satisfies the first part of Definition 1.4. Consider a minimal projective resolution of $R e_{1} R e_{i}$, for some $i, 2 \leq i \leq k$. Since $R e_{1} R e_{i}=E R e_{1} R e_{k}$, a minimal $R$-projective resolution coincides with a minimal $E R E$ projective resolution. Hence the terms of it are in add $\left(R e_{1} \oplus \cdots \oplus R e_{i-1}\right)$. Now consider $R e_{1} R f_{j}$ for some $j, 1 \leq j \leq l$. Once again we have that $R e_{1} R f_{j}=E R e_{1} R f_{j}$, so a minimal projective resolution will have terms from add $\left(R e_{1} \oplus \cdots \oplus R e_{k}\right)$. Hence, $e_{1}$ is a matrix reducing idempotent for $R$.

Next, we need only note that $R / R e_{1} R$ is either $F R F$, if $k=1$, or another ring of the same type as the original. Hence for the first $k-1$ steps, we proceed as above. At this stage, the next factor ring is $F R F$, which has a matrix reducing series, by hypothesis.

Lemma 2.2. Let $R$ be an indecomposable basic left artinian, left serial ring. Then there are orthogonal idempotents $E$ and $F$, with $E+F=1$, so that $F R E=0$, the quiver of FRF is acyclic and the quiver of ERE is a cycle. Moreover, FRF is quasihereditary and if $\mathrm{gl} \operatorname{dim} R<\infty$ then $\mathrm{gl} \operatorname{dim} E F E<\infty$. 
Proof. As in [4] or [6, page 58], the quiver of $R$ is like a rooted tree, with arrows going into the root, where the root may be replaced by an oriented cycle. If the quiver is already acyclic, put $E=0$. Otherwise, let the vertices in the "branches" correspond to the idempotents $e_{1}, \ldots, e_{k}$, and those in the cycle, $e_{k+1}, \ldots, e_{n}$. The simples coming from $e_{1}, \ldots, e_{k}$ do not appear in the projectives $R e_{k+1}, \ldots, R e_{n}$. Put $E=e_{k+1}+\cdots+e_{n}$ and $F=e_{1}+\cdots+e_{k}$. Then $F R E=0$.

Each component of the quiver of FRF has a sink; hence, FRF has a simple projective, $F R e_{i}$ and a heredity ideal $F R e_{i} R F$. Dividing out by $F R e_{i} R F$ gives another ring of the same type. In this way we get a heredity chain for $F R F$.

The last statement follows from [6, Proposition 2.5].

If $R$ is a left serial ring whose quiver is a cycle, then $R$ has a Kupisch series ([2, $\S 32]$ ), and then, for our needs, behaves like a serial ring.

Proposition 2.3. If $R$ is a left serial artinian ring which is of finite global dimension, then $R$ has a left matrix reducing series.

Proof. Assume, as we may, that $R$ is basic and indecomposable. Using the language of (2.2), Proposition 2.1 says that $R$ has a left matrix reducing series if $E R E$ does. Thus we may assume that the quiver of $R$ is a cycle and, hence, that $R$ has a Kupisch series, $R e_{1}, \ldots, R e_{n}$.

Methods and notation from Gustafson [8] will be used, but modified to the left hand version to conform to the conventions of this note. Hence the function $f:\{1, \ldots, n\} \rightarrow\{1, \ldots, n\}$ will here be defined $f(i)=\left[i-c_{i}\right]$, where " $[j]$ " means the least positive residue of $j$ modulo $n$ and $c_{i}$ is the composition length of $R e_{i}$. Put $X=\left\{i \mid 1 \leq i \leq n\right.$ and $f^{s}(i)=i$ for some $\left.s \geq 0\right\}$. It is clear that $f(X) \subseteq X$. By [8, (5)], the minimal projective resolution of $R e_{i} R e_{j}$, insofar as the terms are non-zero, looks like

$$
\cdots \rightarrow R e_{f^{2}(i)} \rightarrow R e_{f^{2}(j)} \rightarrow R e_{f(i)} \rightarrow R e_{f(j)} \rightarrow R e_{i} \rightarrow R e_{i} R e_{j} \rightarrow 0
$$

Also, by [8, (6)], if $i, j \in X$, any homomorphism $g: R e_{j} \rightarrow R e_{i}$ is either 0 or is an isomorphism. Hence, in particular, if $i \in X, \mathrm{LL}\left(R e_{i}\right) \leq n$.

The claim is that if $m \in X$ then there is a matrix reducing series starting with $m$. Let $A_{0}=\left\{i \mid R e_{m} R e_{i}=0\right.$ or $\left.R e_{m} R e_{i} \cong R e_{m}\right\}$, ordered in any way which starts with $m$. Note that $X \subseteq A_{0}$. Next, for any $i \notin A_{0}$, look at a minimal projective resolution of $R e_{m} R e_{i}$ :

$$
\cdots \rightarrow R e_{f(i)} \rightarrow R e_{m} \rightarrow R e_{m} R e_{i} \rightarrow 0
$$

The last non-zero terms are either

$$
0 \rightarrow R e_{f^{k}(m)} \rightarrow R e_{f^{k}(i)} \text { or } 0 \rightarrow R e_{f^{k}(i)} \rightarrow R e_{f^{k-1}(m)}
$$

This means that $f^{k+1}(i)=f^{k+1}(m)$ or $f^{k+1}(i)=f^{k}(m)([8,(3)])$. In either case, $f^{k+1}(i) \in$ 
$X \subseteq A_{0}$. Let the elements of $\left\{i, f(i), f^{2}(i), \ldots\right\} \backslash A_{0}$ be ordered $f^{a_{1}}(i), f^{a_{2}}(i), \ldots, i$, with $a_{1}>a_{2}>\cdots>0$. Add these elements to $A_{0}$, following the elements of $A_{0}$ in the order, to form $A_{1}$. Notice that for any $f^{\prime}(i)$ with $R e_{m} R e_{f(i)} \neq 0$, the minimal projective resolution of $R e_{m} R e_{f^{\prime}(i)}$ has terms with indices in $A_{0}$ or $f^{l+1}(i), f^{l+2}(i), \ldots$; these latter are either among the new indices added to $A_{0}$ or are already in $A_{0}$.

If $A_{1} \neq\{1, \ldots, n\}$, then the process may be repeated by picking $i \notin A_{1}$ and adjoining elements from $\left\{i, f(i), f^{2}(i) \ldots\right\} \backslash A_{1}$ in the same way as in the previous step, to form $A_{2}$. This process continues until the indices are included in some $A_{k}$. Then $A_{k}$ is ordered to form a matrix reducing series for $R$.

Proposition 2.4. Let $R$ be a left serial, left artinian ring. Then the following are equivalent: (a) $R$ is left $k$-hereditary for some $k,(b) R$ is neat, and (c) $\mathrm{gl} \operatorname{dim} R<\infty$.

Proof. Both "neat" and "left $k$-hereditary" imply finite global dimension ([1, Corollary 4] and (1.1)). Now assume $R$ is left serial and of finite global dimension. Then, by (2.3), $R$ has a left matrix reducing series and is $a$ fortior $i$ left $k$-hereditary for some $k$. Moreover, $R$ has a simple $R e / J e$ of projective dimension 1 ([4, Lemma 3]). It is immediate that $e$ is a neat idempotent and $\mathrm{gl} \operatorname{dim}(1-e) R(1-e)<\infty$ by [12, Lemma 2] again. Hence, a neat sequence may be built and $R$ is neat.

The next result shows that for a serial ring $R$, "left 1-hereditary" (in fact an apparently weaker condition) and "quasihereditary" coincide. This is not true for more general rings, as we have seen.

Proposition 2.5. If $R$ is an indecomposable artinian serial ring such that there is $i$, $1 \leq i \leq n$, with $\mathrm{p} \operatorname{dim}_{R} R e_{i} R \leq 1$ and $e_{i} J e_{i}=0$, then $R$ is quasihereditary.

Proof. We may assume that $R$ has no simple projectives. We again use the notation of the proof of (2.3). Let $R e_{1}, \ldots, R e_{n}$ be a Kupisch series for $R$ with $R e_{i}$ of composition length $c_{i}$. If $\mathrm{p} \operatorname{dim}_{R} R e_{i} R=0$ then $R$ is quasihereditary by [3, Proposition 2.3].

Suppose that $\mathrm{p} \operatorname{dim}{ }_{R} R e_{i} R=1$ and that $e_{i} J e_{i}=0$ (the latter is equivalent to $c_{i} \leq n$ ). Then there is some $m>0$ so that $R e_{i} R e_{[i+1]} \cong \ldots \cong R e_{i} R e_{[i+m-1]} \cong R e_{i}$, while $\mathrm{p} \operatorname{dim} R e_{i} R e_{[i+m]}=1$. It follows that $c_{i}<c_{[i+1]}<\cdots<c_{[i+m-1]}$. Thus, renumbering the indices cyclically, we may assume that $R e_{1}, \ldots, R e_{k}$ is a chain of maximal length satisfying $c_{1} \leq n, c_{1}<c_{2} \cdots<c_{k}$ and $\mathrm{p} \operatorname{dim} R e_{1} R e_{[k+1]} \leq 1$.

According to [3, Proposition 2.4], in order to complete the proof we need only show that $c_{[k+1]} \leq k$. But, if this is not the case, there is an exact sequence

$$
0 \rightarrow J^{\prime} e_{1} \rightarrow R e_{1} \rightarrow R e_{1} R e_{[k+1]} \rightarrow 0
$$

with $0 \neq J^{\prime} e_{1}$ projective. Then $J e_{1} \cong R e_{n}$, so $c_{n}<c_{1}<\cdots<c_{k}$ and we have a commutative diagram 


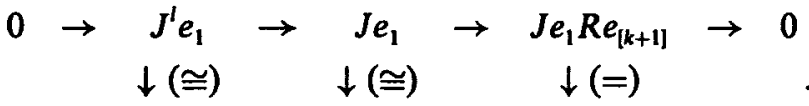

$$
\begin{aligned}
& 0 \rightarrow J^{l-1} e_{n} \rightarrow R e_{n} \rightarrow R e_{n} R e_{[k+1]} \rightarrow 0
\end{aligned}
$$

This contradicts the maximality of $R e_{1}, \ldots, R e_{k}$ since $c_{n}<c_{1}<\cdots<c_{k}$ and $\mathrm{p} \operatorname{dim} R e_{n} R e_{[k+1]}=\mathrm{pdim} R e_{1} R e_{[k+1]}$.

The conclusion of the proposition does not extend to serial rings which are left 2hereditary. The $n=3$ case of the set of examples in [8] is serial of global dimension four. It is left (and right) 2-hereditary but not quasihereditary. (This family is discussed in more detail in (3.3).) For serial rings, global dimension 3 implies quasihereditary by [3, Proposition 2.7] or [10, Theorem 3.2].

\section{Examples}

In the following examples, the diagram notation of [7] is used freely. All algebras are assumed to be over some field $F$.

Example 3.1. There are algebras $R$ which have a left but no right matrix reducing series. Two examples are presented, the first of infinite global dimension, the second of global dimension 4 .
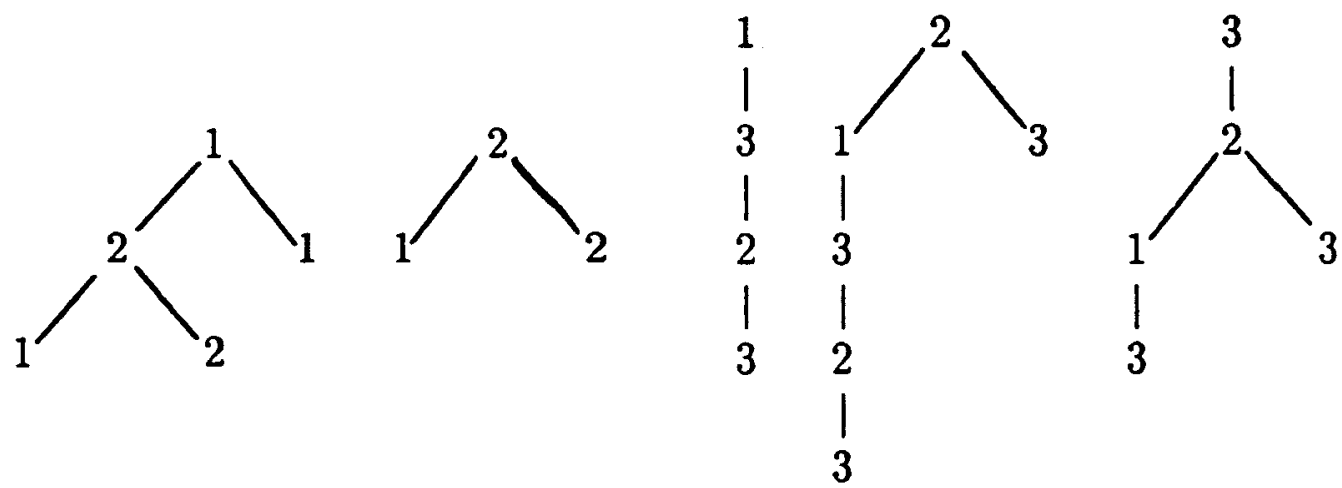

The first algebra has a left matrix reducing series, $e_{2}, e_{1}$, where the ideals to be used are, in fact, projective. However, both $R e_{1} R_{R}$ and $R e_{2} R_{R}$ are of infinite projective dimension. The second algebra has a left matrix reducing series, $e_{1}, e_{2}, e_{3}$ but no right matrix reducing series (it is left and right 2-hereditary).

Example 3.2. There is an algebra $R$ which is left 1-hereditary (and, hence, has a 
matrix reducing series) but not right 1-hereditary. This algebra is right 2-hereditary. Moreover, it is not neat, in the sense of [1].
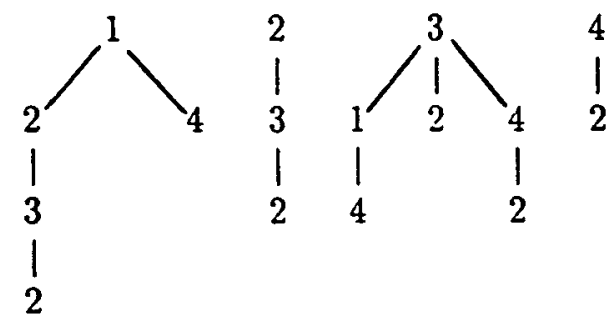

The global dimension is 5 . A left matrix reducing series is $e_{1}, e_{4}, e_{3}, e_{2}$. A right matrix reducing series is $e_{4}, e_{3}, e_{1}, e_{2}$.

While every left artinian ring of global dimension 2 is quasihereditary, one of global dimension 3 need not be even left 1-hereditary. The example [10, (2) page 172] is of global dimension 3; it is left and right 2-hereditary, but neither left nor right 1hereditary. It has a matrix reducing series on each side and is also neat.

Example 3.3. For any $n>2$ there is a basic serial algebra $R$ with $n$ primitive idempotents, global dimension $2 n-2,(2 n-4)$-hereditary but not $k$-hereditary for any $k<2 n-4$.

This can be done by letting the composition length of $R e_{i}$ be $n+1$ for $i=1, \ldots, n-1$ and $R e_{n}$ of composition length $n$. Then the only candidate for a first idempotent in Definition 1.2 is $e_{n}$ and $\mathrm{p} \mathrm{dim}{ }_{R} R e_{n} R=2 n-4$. The series $e_{n}, e_{1}, \ldots, e_{n-1}$ is matrix reducing. This is the family of examples of [8], which exhibit maximal possible global dimension for a serial ring with $n$ idempotents.

We conclude with a method for building a new ring with left matrix reducing series from existing ones.

Example 3.4. Suppose $C$ and $D$ are left artinian rings which have left matrix reducing series, and that $\mathrm{gl} \operatorname{dim} C<\infty$. Suppose also that ${ }_{c} M_{D}$ is a bimodule such that ${ }_{C} M$ is finitely generated. Then $R=\left(\begin{array}{cc}D & 0 \\ M & C\end{array}\right)$ has a left matrix reducing series.

This is a corollary of Proposition 2.1. The easiest example is to start with a left artinian ring $A$ of finite global dimension which has a left matrix reducing series. Then $R=\left(\begin{array}{cc}A & 0 \\ A & A\end{array}\right)$ also has one. 


\section{REFERENCES}

1. I. Agoston, V. Dlab and T. Wakamatsu, Neat algebras, Comm. Algebra 19 (1991), 433-442.

2. F. W. ANDERSON and K. R. Fuller, Rings and Categories of Modules, second edition (Springer-Verlag, New York, Heidelberg, Berlin, 1992).

3. W. D. Burgess and K. F. Fuller, On quasihereditary rings, Proc. Amer. Math. Soc. 106 (1989), 321-328.

4. W. D. Burgess, K. R. Fuller, E. Voss and B. Zimmermann-Huisgen, The Cartan matrix as an indicator of finite global dimension for artinian rings, Proc. Amer. Math. Soc. 95 (1985), 157-165.

5. V. Dlab and C. M. Ringel, Quasi-hereditary algebras, Illinois J. Math. 33 (1989), 280-291.

6. K. R. Fuller, The Cartan determinant and global dimension of artinian rings, Contemp. Math. 124 (1992), 51-72.

7. K. R. Fuller, Algebras from diagrams, J. Pure Appl. Algebra 48 (1987), 23-37.

8. W. Gustafson, Global dimension in serial rings, J. Algebra 97 (1985), 14-16.

9. M. Hoshino and Y. Yukimoto, A generalization of heredity ideals, Tsukuba J. Math. 14 (1990), 423-433.

10. M. Uematsu and K. Yamagata, On serial quasi-hereditary rings, Hokkaido Math. J. 19 (1990), 165-174.

11. K. Yamagata, A reduction formula for the Cartan determinant problem for algebras, Arch. Math. 61 (1993), 27-34.

12. D. ZaCharia, On the Cartan matrix of an artin algebra of global dimension two, $J$. Algebra 82 (1983), 353-357.

Department of Mathematics and Statistics UNIVERSITY OF OTTAWA

Ottawa, Canada K1N 6N5

E-mail address: wdbsg@uottawa.ca
Department of Mathematics

UNIVERSITY OF IOWA

IOWA CITY, IA, USA 52242

E-mail address: kfuller@math.uiowa.edu 